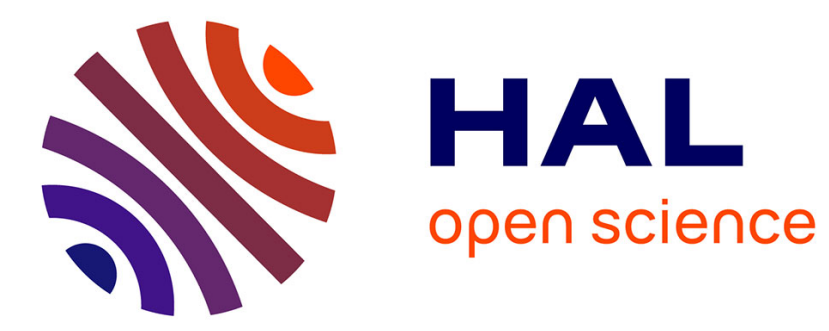

\title{
Delayed Fracture in Porous Media
}

Noushine Shahidzadeh-Bonn, Philippe Vié, Xavier Chateau, Jean-Noël Roux, Daniel Bonn

\section{To cite this version:}

Noushine Shahidzadeh-Bonn, Philippe Vié, Xavier Chateau, Jean-Noël Roux, Daniel Bonn. Delayed Fracture in Porous Media. Physical Review Letters, 2005, 95 (17). hal-01982951

\section{HAL Id: hal-01982951 https://hal.science/hal-01982951}

Submitted on 17 Jan 2019

HAL is a multi-disciplinary open access archive for the deposit and dissemination of scientific research documents, whether they are published or not. The documents may come from teaching and research institutions in France or abroad, or from public or private research centers.
L'archive ouverte pluridisciplinaire HAL, est destinée au dépôt et à la diffusion de documents scientifiques de niveau recherche, publiés ou non, émanant des établissements d'enseignement et de recherche français ou étrangers, des laboratoires publics ou privés. 


\title{
Delayed Fracture in Porous Media
}

\author{
Noushine Shahidzadeh-Bonn, ${ }^{1}$ Philippe Vié, ${ }^{1}$ Xavier Chateau, ${ }^{1}$ Jean-Noël Roux., and Daniel Bonn ${ }^{2,3}$ \\ ${ }^{1}$ Institut Navier, Laboratoire dès Matériaux et Structures du Génie Civil, 2 allée Kepler, 77420 Champs sur Marne, France \\ ${ }^{2}$ Laboratoire de Physique Statistique, Ecole Normale Supérieure, 24 rue Lhomond, 75231 Paris Cedex 05, France \\ ${ }^{3}$ Van der Waals-Zeeman Instituut, University of Amsterdam, Valckenierstraat 651018 XE Amsterdam, The Netherlands
} (Received 16 February 2005; published 17 October 2005)

The fracture of porous media subjected to a constant load is studied. Contrary to homogeneous solids in which fracture usually happens instantaneously at a well-defined breaking strength, the fracture of a porous medium can occur with a delay, allowing us to quantify the average lifetime of the unbroken material. We show that the average fracture probability, a key property for risk analysis in civil engineering, is given by the probability of crack nucleation. The nucleation process can be understood qualitatively by calculating the activation energy for crack nucleation, taking into account the porosity of the medium.

DOI: 10.1103 /PhysRevl.ett. $95.17550 \mathrm{l}$

PACS numbers: $62.20 \mathrm{Mk}, 46.50 .+\mathrm{a}$

The failure of porous materials, despite its great importance for both civil engineering (concrete being a porous medium) and oil recovery, is still ill understood [1-4]. Usual fracture experiments either determine a fracture threshold stress of a certain material or study fatigue, fracture due to the application of a cyclic load [1,2]. However, the key quantity for risk analysis in civil engineering and profitability in oil recovery is the probability that a given porous medium fractures under a given load.

From a more fundamental point of view, the effect of disorder on the fracture strength of heterogeneous materials is incompletely understood [5]. The failure of such materials can only very rarely be related directly to the mechanical properties of the material, but is mostly due to the presence of defects, causing the material to fail at its weakest points. The fracture of such strongly disordered materials is usually discussed in terms of failure distributions (e.g., the Weibull distribution), giving the probatbility of failure of a sample of a given size under a given stress. Our theoretical understanding of this problem has greatly improved in recent years by applying ideas of statistical mechanics [5]. However, for weak disorder, practically the more relevant case [5], many important questions remain to be answered. Recent experimental activity has focused on performing well-controlled experiments on some model heterogeneous materials [6]. In these systems, the fracture can occur with a delay; the delay has been associated with the existence of an energy barrier for nucleating the initial crack. Theory has provided us with different predictions for the energy barrier, and hence for the lifetime of the unbroken material [7]. However, experimentally the disorder could not be varied a systematic way so that its effect on the failure probability remains unknown.

In this Letter we study the fracture properties of porous materials of controlled disorder, and show that a direct relation between fracture and material properties can be established. For this, we manufacture porous media of controlled porosity. Additionally, our experimental frac- ture geometry (three-point flexion) imposes the exact location of the fracture. This prevents the material from nupturing at its weakest point, and consequently allows us to relate the fracture properties to the measured bulk elastic properties. In agreement with theory $[6,7]$ we find that the fracture of porous media is a stochastic phenomenon. We thus study the time delay to the spontaneous failure of porous media of controlled porosity subject to a permanent load. The energy for the spontaneous nucleation of the initial fracture determines the lifetime of the unbroken material, and can be related directly, through its bulk elastic properties, to the structure (porosity) of the material.

The model porous material we use in ourexperiments is made of sintered glass beads of diameter $104-125 \mu \mathrm{m}) \mathrm{We}$ devised a novel method allowing one to control the porosity $\phi$ in the range $20 \%<\phi<50 \%$. Glass beads are mixed with equal-sized salt grains $(\mathrm{NaCl})$ in varying proportion,

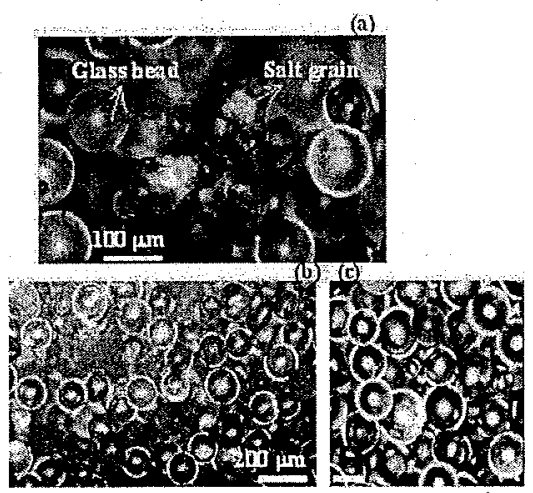

FIG. 1. Picture of the porous medium (consolidated glass bead). The porosity is determined using two techniques: imbibition to water and gamma densitometry. (a) Salt grains trapped in the consolidated material. (b) Porous medium after dissolving the salt $\phi=0.48$. (c) Porous material without salt $\phi=0.3$. 
and packed in a mold. The sintering process consists in heating the packing near the glass transition temperature $\left(630^{\circ}-650^{\circ}\right)$, so that the glass beads soften and stick together (but not the salt grains, which melt at $800^{\circ} \mathrm{C}$ ), forming solid bridges of diameter $d \approx 28 \mu \mathrm{m}$. Upon cooling, they form a consolidated porous material (Fig. 1). Subsequently, water is used to dissolve the trapped salt grains. Since the salt grains were distributed randomly, this introduces a random disorder in the material. Porosities of up to $50 \%$ were obtained [Fig. 1(c)]; samples were cut into $(9 \times 80 \times(1.6 \mathrm{~mm})$ test bars.

The three-point flexion experiments (Fig. 2) show that upon increasing the force $F$ applied to the middle of the bar, we first observe an almost perfect elastic response [Fig. 3(a)], allowing for the evaluation of the Young's modulus $E$ of the materials. Measuring $E$ for samples with a different porosity, we find a linear dependence to within the experimental accuracy [Fig. 3(b)].

To understand the dependence of the Young's modulus on the porosity shown in Fig. 3(b), we consider a predictive continuum micromechanical model for the linear elasticity of our porous medium [8]. Different models exist for heterogeneous solids; the so-called self-consistent approximation we use here is the simplest one. It assumes that the medium is made of two different types of spherical inclusions (the glass beads and the empty pores), randomly distributed over the sample. The material outside each inclusion is assumed homogeneous so that macroscopically, the material is isotropic. The dependence of the macroscopic Young's modulus on the porosity $\phi$ can be calculated analytically, and is given by

$$
E=E_{0}\left[\frac{20 \nu_{0}+63 \phi^{2} \nu_{0}-69 \phi \nu_{0}-27 \phi^{2}+15 \phi+8+(3 \phi-4) \sqrt{\Delta}}{10\left(2 \nu_{0}-1\right)\left(3 \phi \nu_{0}-3 \phi+2\right)}\right]
$$

where $E_{0}$ and $\nu_{0}$ denote, respectively, the Young modulus and the Poisson ratio of the glass beads and

$$
\begin{aligned}
\Delta= & 49-114 \phi+25 \nu_{0}^{2}+81 \phi^{2}+336 \phi \nu_{0}-270 \phi \nu_{0}^{2} \\
& -378 \phi^{2} \nu_{0}-70 \nu_{0}+441 \phi^{2} \nu_{0}^{2} .
\end{aligned}
$$

Although this equation is rather complicated, we find that the result for $E(\phi)$ is very well approximated (numerically to within $5 \%$ ) by the simple expression $E \approx E_{0}(1-2 \phi)$, independently of the value of $\nu_{0}$ [drawn line in Fig. 3(b)] [9]. We have not been able to find an analytical justification of this important simplification. The only adjustable parameter is $E_{0}=2.2 \times 10^{10} \mathrm{~Pa}$, a reasonable value in view

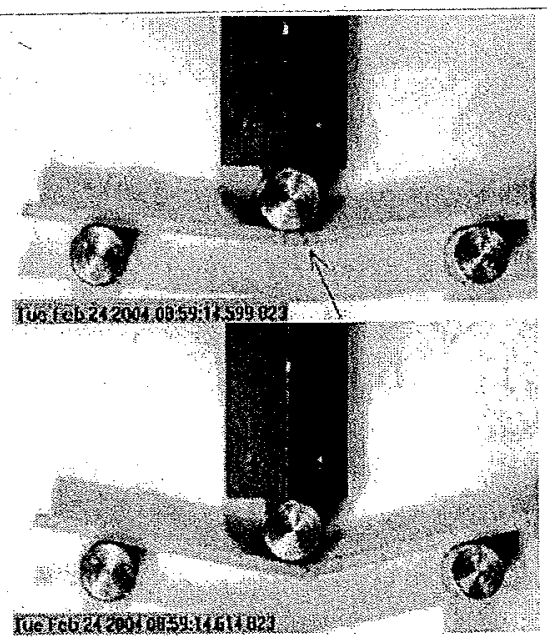

FIG. 2. The three-point flexion experiment. Pictures taken with a high-speed camera operating at 20000 frames/s at the moment of rupture.
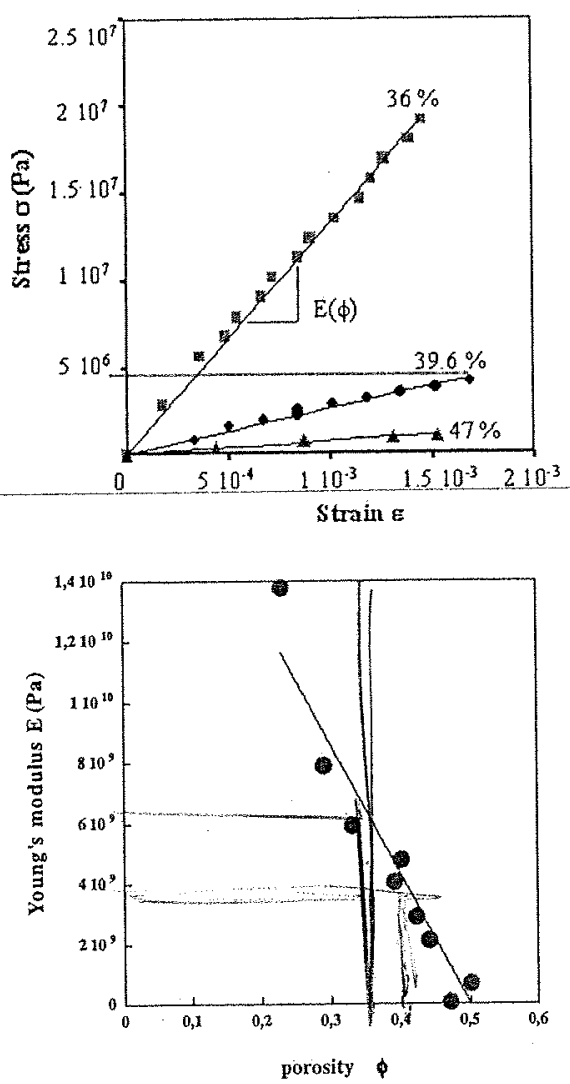

FIG. 3 (color online). (a) Stress vs deformation curve in the three-point flexion experiment; tesults for samples of porosities $0.36,0.396$, and 0.47 . (b) Linear dependence of the Young modulus versus porosity, $E_{0}=2.2 \times 10^{10} \mathrm{~Pa}$. 
of the simplifications made here; $E_{\text {glass }}=7 \times 10^{10} \mathrm{~Pa}$. The difference probably originates from the assumptions of the model; first, although it is reasonable to assume that the solid beads remain spherical in the model porous medium, this assumption is clearly highly questionable for the voids. Second, the self-consistent model does not account rigorously for the number of bonds between glass beads. In spite of these shortcomings, it describes the experimental observations well, and will be very useful for understanding the fracture experiments.

The fracture experiments were done in the same threepoint flexion setup. When a constant force $F$ is applied, the breaking does not occur instantaneously but happens after a certain delay time. For a given force, the distribution of lifetimes is a Poisson distribution [Fig. 4(b)]. This demonstrates the random nature of the fracture process, and allows one to define a mean breaking time $t_{b}$. Changing the force, $t_{\mathrm{b}}$ shows an extremely steep function of the stress
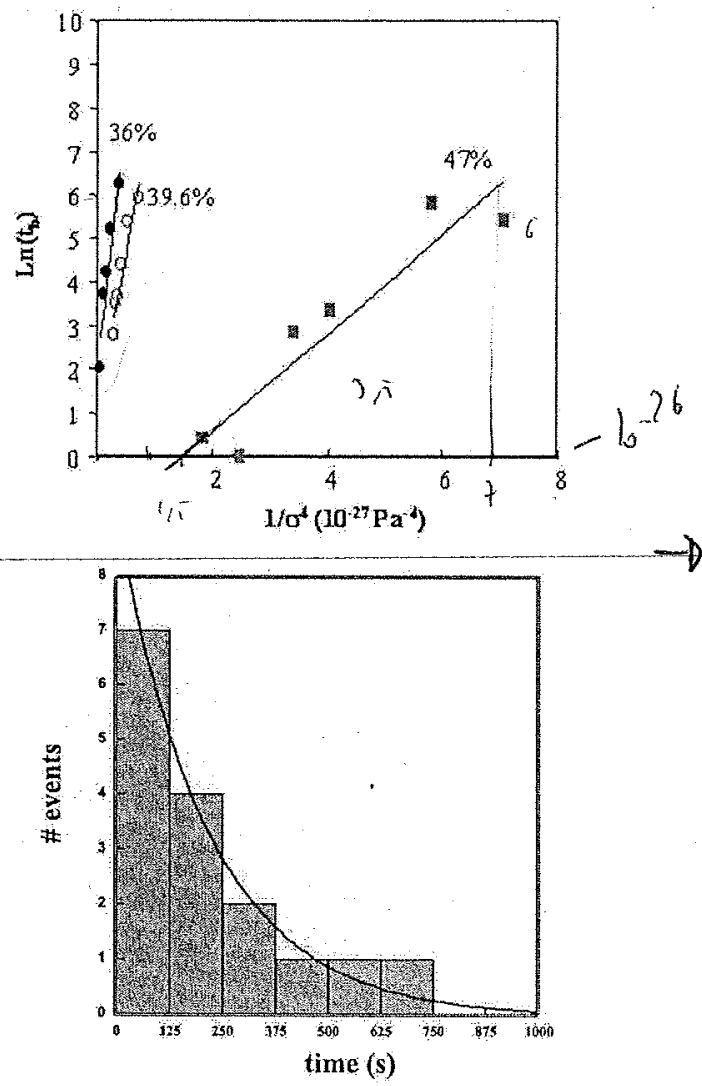

FIG. 4 (color online). (a) The apparent activation energy (In of the breaking time/s) as a function of the maximum stress to the fourth power. A linear dependence is observed. (b) Distribution of the breaking times $t_{\mathrm{b}}$ for a large number of experiments at a constant force and for a porosity of $40 \%$; the drawn line is a Poisson distribution. $\sigma$ [Fig. 4(a)]: changing the stress $\sigma$ by a factor of $2, t_{\mathrm{b}}$ can vary by almost 2 orders of magnitude. Each data point for a given porosity and stress corresponds to an average of at least 15 experiments. For all of these series of experiments, the delay times are Poisson distributed. This directly implies that the standard deviation is rather large, reflecting the random nature of the fracture process; the error on $t_{\mathrm{b}}$ is typically half the lifetime itself.

If an energy barrier for nucleating a crack exists, and the nucleation is thermally activated, $t_{\mathrm{b}}$ should be inversely proportional to the nucleation or fracture probability $p \sim$ $\exp \left(-U_{\text {act }} / k_{B} T\right)$; the lifetime $t_{\mathrm{b}}$ of the unbroken material is then inversely proportional to the nucleation probability. Griffith $[2,10]$ evaluated $U_{\text {act }}$ for crack nucleation as the result of a competition between the cost in fracture (surface) energy $U_{s}$ due to the crack growth and the gain in elastic (volume) energy $U_{\mathrm{e}}$ due to stress relaxation in the vicinity of the crack. Applying such ideas to a small crack surface of extension $L$ and surface $L^{2}[6,7]$, one gets up to factors of order unity $U_{s} \approx \Gamma L^{2}$. Here $\Gamma$ is the fracture energy; $\Gamma$ is different from the surface tension to indicate that the process is irreversible: once broken, the material cannot be healed by simply bringing the two pieces back into contact. This irreversibility is usually assumed to be also incorporated in the fracture energy [1-4]. For the elastic energy, $U_{e} \approx \sigma^{2} L^{3} / E$; this follows from the fact that the elastic energy density $\sigma^{2} / 2 E$ in a medium of Young's modulus $E$ and under a uniform stress $\sigma$ is gained in a volume $\approx L^{3}$. Maximizing $U_{\mathrm{s}}-U_{\mathrm{c}}$ yields $U_{\text {act }} \approx$ $\Gamma^{3} E^{2} / \sigma^{4}$ and the critical crack length $L_{\mathfrak{c}} \approx \Gamma E / \sigma^{2}$. We therefore plotted $\ln \left(t_{\mathrm{b}}\right) \sim U_{\text {act }}$ as a function of $\sigma^{4}$ [Fig, 4(a)]; although our experimental window is limited due to the steep dependence of $t_{\mathrm{b}}$ on $\sigma$, the prediction agrees quantitatively with our experiments. If we take $E=$ b) $7 \times 10^{10} \mathrm{~Pa}, \mathrm{~F}=10 \mathrm{~J} / \mathrm{m}^{2}$, and $\sigma=50 \times 10^{7} \mathrm{~Pa}$, we ob-

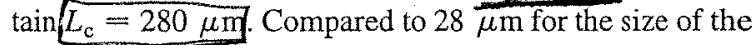
bridges between neighboring particles, there is a sufficient separation of length scales to consider the material homogeneous at the scale for the initial fracture, a prerequisite for our Griffith-like theory to be applicable.

The question is then how to relate $U_{\text {act }}$ to the porosity $\phi$. For this it is sufficient to realize that in our experiments, $\Gamma$ depends on $\phi$ in the same way as the Young's modulus $E$. This is because both $E$ and $\Gamma$ are determined by the number of contacts between touching spheres, and in order to create the fracture surface one has to remove these contacts. Putting in the approximation for $E$ discussed above yields $U_{\text {act }} \sigma^{4} \approx \Gamma^{3} E^{2} \propto(1-2 \phi)^{5}$ which extends Griffith's theory to porous media. This new expression is in excellent agreement with the experiments (Fig. 5): we thus obtained a direct relation between the porosity and the time after which the structure fails.

Two caveats are the following: First, since we have not done experiments as a function of temperature, we cannot confirm that the process is really an activated one. 


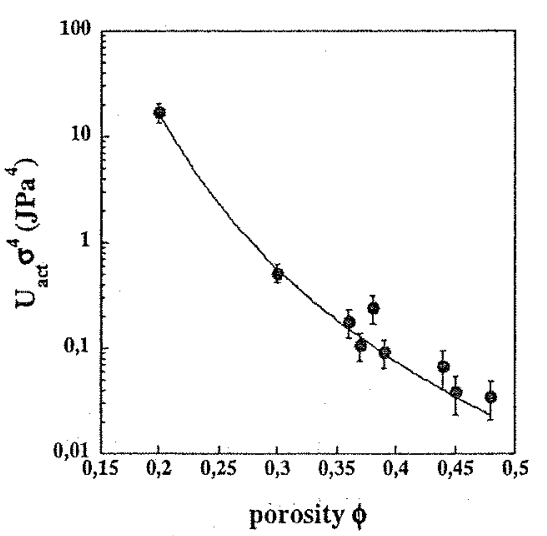

FIG. 5 (color online). The slope of Fig. 4 as a function of the porosity. The drawn line is the prediction from the scaling arguments presented in the text.

Additionally, application of thermodynamic arguments to fracture nucleation is controversial, since it does not take into account the irreversible components of the fracture process. We have incorporated these irreversible components into the fracture energy $\Gamma$. We do, however, confirm that the delay is a consequence of a nucleation process of the first critical crack and agrees with the Griffith theory. This follows (i) from the random nature of the fracture process and (ii) from the observation that the logarithm of the lifetime varies as the inverse fourth power of the stress. Second, plugging in typical values for $\sigma, \Gamma$, and $E$, the activation energy is found to be on the order of $10^{-12} \mathrm{~J}$, much larger than $k_{B} T$. There can be two reasons for this discrepancy. One possible reason could be that $\Gamma$ is much smaller than that of bulk glass, because the sintering leads to a relatively weak plane in the solid bridges. A measurement of the fracture energy using the notch method [11] yields $\Gamma=5 \pm \mathrm{Jm}^{-2}$, close to the fracture energy of glass; this therefore does not seem a plausible explanation for the observed discrepancy. A more likely explanation is that the irreversibility of the (micro) fracture process leads to an important difference with classical reversible nucleation processes [7]. A small crack formed due to a fluctuation will not disappear, and is a favorable starting point for a further growth. Consequently, the existence of unhealed microcracks leads to weaker regions, which accelerates the failure. Also the heterogeneity leads to stress concentration in the material, and so the local stress is larger than the macroscopic one. Since the barrier varies as $1 / \sigma^{4}$, this is a sizable effect. This is, in fact, well known for heterogeneous materials, and was also observed in other experiments and in model calculations [6,7]: the disorder lowers the barrier by an important amount. Therefore both the irreversibility of the fracture process and the concentration of the stress may provide a possible explanation for the large difference between the activation energy calculated with the macroscopic parameters and $k_{B} T$.

In conclusion, we have demonstrated that a direct relation between fracture probability and the structural and mechanical properties of a disordered material can be established. These results should not only be relevant for the civil engineering industry, in which porous media are widely used, and for oil recovery but could also have implications for the strength of composite materials.

LPS de l'ENS is UMR 8550 of the CNRS, associated with the Universities Paris VI et VII. LMSGC is UMR 113 of the CNRS, associated with the LCPC and the ENPC.

[1] B.R. Lawn, Fracture of Brittle Solids (Cambridge University Press, Cambridge, 1975)

[2] L. B. Freund, Dynamic Fracture Mechanics (Cambridge University Press, Cambridge, 1990)

[3] M.F. Kanninen and C.H. Popelar, Advanced Fracture Mechanics (Clarendon, Oxford, 1985).

[4] M. Marder and J. Fineberg, Phys. Today 49, No. 09, 24 (1996).

[5] B. K. Chakrabarti and L. G. Benguigui, Statistical Physics of Fracture and Breakdown in Disordered Systems (Clarendon, Oxford, 1997).

[6] L. Pauchard and J. Meunier, Phys. Rev. Lett. 70, 3565 (1993); D. Bonn, H. Kellay, M. Prochnow, K. BenDjemiaa, and J. Meunier, Science 280, 265 (1998); A. Guarino, S. Ciliberto, and A. Garcimartin, Europhys. Lett. 47, 456 (1999); S. Santucci, L. Vanel, and S. Ciliberto, Phys. Rev. Lett. 93, 095505 (2004).

[7] L. Gubolovic and S. Feng, Phys. Rev. A 43, 5223 (1991); Y. Pomeau, C.R. Acad. Sci., Ser. II 314, 553 (1992); S. Roux, Phys. Rev. E 62, 6164 (2000); S. Santucci, L. Vanel, A. Guarino, R. Scoretti, and S. Ciliberto, Europhys. Lett. 62, 320 (2003); S. Ciliberto et al., Physicá-Átusterdam) 1580,-83 (2001).

[8] M. Marder, Nature (London) 381, 275 (1996); S. NematNasser and M. Hori, Micromechanics: Overall Properties of Heterogeneous Materials (North-Holland, Amsterdam, 1993); S. Torquato, Random Heterogeneous Materials: Microstructure and Macroscopic Properties (Springer, New York, 2002).

[9] $E$ is nearly independent of $\nu_{0}$ because the material is porous. When submitted to a uniaxial stress, lateral strains induced by the Poisson effect can develop freely at the microscopic scale thanks to the available porous space. This means that only negligible lateral stresses appear at the microscopic level and the macroscopic Young's modulus is nearly independent of the microscopic Poisson ratio.

[10] A. A. Griffith, Phil. Trans. R. Soc. A 221, 163 (1920).

[11] Notches of different lengths $(0.1-1 \mathrm{~mm})$ were machined into $3 \mathrm{~mm}$ thick bars of $30 \%$ porosity. The critical stress for fracture was measured and allows us to determine the critical stress intensity factor at fracture. From the variation of the stress intensity factor with the length of the notch, the fracture energy can be calculated. 